\title{
El estudio de la economía gremial. El caso del sindicato argentino Luz y Fuerza Capital Federal (1943-2003)
}

\author{
The study of the union economy. The case of the \\ Argentinian guild Luz y Fuerza Capital Federal \\ (1943-2003) \\ doi: http://dx.doi.org/10.32870/ \\ Julieta Haidar

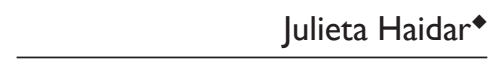

espiral.v24i70.6432

\section{Resumen}

Este artículo identifica e interpreta las continuidades y transformaciones producidas en los mecanismos reproductivos de un sindicato en distintas etapas históricas, bajo la hipótesis de que la economía gremial es de vital importancia para entender las dinámicas y estrategias de las organizaciones sindicales. Se analizan el concepto de mecanismos sindicales de reproducción material y tres dimensiones: el nivel de complejidad, sus efectos sobre la estructura organizativa y patrimonial del gremio, y su relación con la economía política de los trabajadores. Se efectúa un estudio de caso en profundidad del sindicato Luz y Fuerza Capital Federal (LYFCF) de Argentina, esto en tres periodos (1943-1976, 1976-1992, 1992-2003). Se concluye que existe un vacío analítico sobre la economía gremial,y que esta dimensión es relevante en la construcción de un sindicato y en la definición de sus estrategias.

Palabras clave: sindicalismo, economía gremial, mecanismos sindicales de reproducción material,Argentina, Luz y Fuerza Capital Federal.

\begin{abstract}
This article identifies and interprets the continuities and transformations in the material reproduction mechanisms of a union at different historical stages, under the hypothesis that the union economy is vital to understand the dynamics and strategies of trade unions. We use the concept of union material reproduction mechanisms and we analyze three dimensions: the level of complexity, its effects on organizational and patrimonial structure and its relationship with the workers political economy. We perform a case study in depth: the guild of Luz y Fuerza Capital Federal (LYFCF) of Argentina in three historical stages (19431976, 1976-1992, and 1992-2003). This article concludes that there is an analytical vacuum about union economy, and that this dimension is relevant in any union building and strategies defining.
\end{abstract}

Keywords:trade unionism, union economy, union material reproduction mechanisms, Argentina, Luz y Fuerza Capital Federal.

-Profesora-Investigadora de la Universidad de Buenos Aires (UBA), CONICET, Argentina. julietahaidar@yahoo.com.ar

Fecha de recepción: 06 de enero de 2017. Fecha de aceptación: 02 de junio de 2017. 


\section{Introducción}

Esta investigación se interesa por el sindicalismo, una fuerza social que constituye también un hecho central de la vida social y política en Argentina. Dada su relevancia, los sindicatos han sido estudiados desde múltiples núcleos problemáticos y disciplinas. Sin embargo, no se analizaron antes desde una dimensión asociada a la economía gremial.

Este artículo se propone contribuir a saldar este vacío con una propuesta teórica y empírica que estudia los mecanismos sindicales de reproducción material (MSRM), esto es, los mecanismos que permiten a los sindicatos reproducirse económicamente (Haidar, 2015c).

Este estudio es pertinente porque, en primer lugar, existe un vacío analítico respecto a la economía gremial, lo que convierte a esta dimensión en objeto de interés. En segundo término, contribuye a pensar cuál es la relevancia de la dimensión económica en la construcción de un sindicato y en la definición de sus estrategias. En tercer lugar, el estudio de la reproducción de un gremio sienta las bases para entender los vínculos materiales que se establecen entre la organización y los trabajadores a los que representa, ya que, como se demostrará, los mecanismos sindicales de reproducción material pueden estar ligados o disociados de la dinámica reproductiva de los asalariados.

Con este horizonte de posibilidades, el objetivo específico de la investigación es identificar e interpretar las continuidades y transformaciones producidas en los mecanismos de reproducción material de un sindicato en distintas etapas, bajo la hipótesis de que la economía gremial es de vital importancia para entender las dinámicas y estrategias de las organizaciones sindicales.

Para avanzar en este sentido, se revisa la literatura que estudia el sindicalismo y se construye un marco interpretativo que permite analizar los mecanismos reproductivos 
de los gremios. Luego, se aplica ese marco para estudiar en profundidad el caso del sindicato Luz y Fuerza Capital Federal (LYFCF) de Argentina en tres etapas históricas: 1943-1976, 1976-1992 y 1992-2003.

La pertinencia del caso está fundada en los siguientes aspectos: 1) LYFCF es el mayor gremio argentino de uno de los sectores más importantes de la economía, el eléctrico, lo que lo vincula políticamente a la federación de sindicatos de trabajadores eléctricos de Argentina (cuyo nombre oficial es Federación Argentina de Trabajadores de Luz y Fuerza, FATLYF); 2) dicho sindicato alcanzó un alto nivel de desarrollo organizativo y patrimonial, situación que le valió un lugar protagónico en el mapa sindical argentino, lo que se fue modificando en distintas etapas históricas; 3) por último, posee un rico cuerpo documental que permite construir el corpus de la investigación (es decir, convenios colectivos de trabajo, estatutos sindicales, revistas Dinamis, todo entre 1943 y 2003).

\section{La problematización en torno a los sindicatos y la economía gremial'}

Los sindicatos han sido estudiados desde diferentes disciplinas y perspectivas, las cuales definieron como objeto de análisis a un conjunto vasto y heterogéneo de problemas. Una reconstrucción exhaustiva de estos excede los objetivos del presente trabajo. No obstante, es posible reconocer algunos de los temas que aparecen en forma recurrente dentro de la literatura especializada.

Uno de ellos es la relación entre los sindicatos y el Estado. Se trata de una problemática estudiada desde enfoques institucionalistas y que tiene como objetos principales los niveles de autonomía y la capacidad de presión que tienen 
las organizaciones sindicales en relación con el Estado (Schmitter, 1992; Korpi, 1985; Collier y Collier, 1991, entre otros). Este tema también es analizado desde las teorías del corporativismo y los recursos de poder: la primera ve a los sindicatos como grupos de interés que inciden en el proceso de definición de políticas económicas o sociales, mientras que la segunda aporta que para esa definición las organizaciones cuentan con distintos recursos de poder (Moe, 1980; Robinson, 1998; Lévesque y Murray, 2004).

En esta misma línea, en Argentina gran parte del análisis sobre sindicalismo se centra en la relación entre los sindicatos, el Estado y los partidos políticos (Bunel, 1992; Fernández, 2002; Levitsky, 2005); los estudios realizados sobre las estrategias sindicales frente a las reformas de mercado de los años noventa son una expresión de esta perspectiva (Murillo, 1997; Etchemendy y Palermo, 1998; Senén González y Bosoer, 2009; Etchemendy, 2001, por referir algunos).

Asimismo, en sintonía con la problematización sobre la relación entre el Estado y los sindicatos, se ubican gran parte de las producciones historiográficas sobre el surgimiento y desarrollo del peronismo. Con múltiples matices en cuanto a enfoques teóricos e intereses analíticos, pueden rastrearse aquí trabajos clásicos como los de Del Campo (1983), Doyon (1984), Torre (1990), Murmis y Portantiero (1987), y James (1999), entre muchos otros.

Otro tema estudiado en torno al sindicalismo versa sobre la dupla democracia-burocracia sindical. Este se interesa por los procesos políticos internos de las organizaciones gremiales, la conformación y desarrollo de la representación en los lugares de trabajo y la relación entre dirigentes y bases. En el ámbito internacional, se destacan las investigaciones clásicas de Michels (2010), Lipset (1963), Mandel (1987), y Stepan-Norris y Zeitlin (1995); en Latinoamérica, algunas producciones que recuperan y avanzan en estos debates 
son Bensusán (2000), De la Garza (2001), Zapata (2004) y la revista Nuevo Topo (2010).

Por otro lado, en Argentina abundan los trabajos historiográficos sobre las luchas de sindicatos clasistas frente a la burocracia sindical (Gordillo, 1991; Löbbe, 2006; Schneider, 2007), así como estudios de caso sobre las dinámicas de organización de nuevos colectivos, comisiones internas y líneas opositoras (Battistini, 2004; Montes Cató y Ventrici, 2008; Abal Medina y Diana Menéndez, 2011).

Al mismo tiempo, vinculado a este último debate, encontramos un conjunto basto de producciones que problematizan la relación entre el sindicalismo y la izquierda, entre ellas las obras clásicas de Marx y Engels, Lenin, Michels, Trotsky, Losovsky y Gramsci, que discuten el potencial revolucionario del sindicalismo en una sociedad capitalista. Con posterioridad, esta problemática fue recuperada en las producciones de Hyman (1978), Mandel (1973) y Lapides (1987), en Cuadernos de pasado y presente (1978), entre otras. En Argentina, cercano a esta discusión, hay un interés historiográfico por la relación entre el movimiento obrero y la izquierda (Falcón, 1984; Suriano, 1990; Iñigo Carrera, 2004; Camarero, 2007, entre muchos otros).

Esta breve descripción dista mucho de reconstruir todos los estudios sobre sindicalismo, pero evidencia que los dedicados a analizar los procesos internos de los sindicatos son poco numerosos, y que los existentes privilegian aspectos organizativos, institucionales, identitarios y culturales, y dejan casi inexplorada la dimensión asociada a la economía gremial.

En ese marco, el presente artículo se presenta como una contribución para saldar ese vacío y propone estudiar la economía gremial a través del concepto de los MSRM, definidos como "aquellos mecanismos que expresan las relaciones sociales a través de las cuales las organizaciones gremiales se reproducen económicamente" (Haidar, 2015c, p. 26). 
Este concepto: 1) alude en forma específica a los modos en que los sindicatos sostienen y expanden su economía, es decir, supone una noción ajustada de materialidad que excluye aspectos discursivos, identitarios u organizacionales, 2) asocia la economía a relaciones sociales, con lo cual se aparta de una perspectiva economicista, descosifica la problemática y la sitúa en una dimensión productiva y política, bajo la idea de que la materialidad de un gremio expresa relaciones sociales de lucha.

Por lo tanto, analizar el desarrollo de la economía gremial a la luz del concepto de MSRM o, más sintéticamente, "mecanismos reproductivos", no significa auditar los ejercicios económicos de un sindicato, sino establecer conexiones teóricas y movimientos tendenciales que permitan identificar las continuidades y transformaciones en sus principales características. Para avanzar en este sentido, se propone clasificar los mecanismos reproductivos según tres dimensiones:

a) El nivel de complejidad: las relaciones sociales pueden ser simples o más complejas.

b) Los efectos sobre la estructura organizativa y patrimonial del gremio: los efectos de los mecanismos reproductivos pueden ser expansivos o contractivos y encontrar su fundamento en el consumo o en la valorización.

c) La relación con la economía política de los trabajadores: los mecanismos reproductivos pueden estar más o menos articulados con la economía política de los trabajadores.

Los conceptos utilizados en esta clasificación encuentran inspiración en algunas de las categorías desarrolladas en $E l$ Capital de Marx. Sin embargo, no se pretende aplicarlos en forma estricta, lo que resultaría imposible dado que: 1) estas categorías están reservadas al estudio del capital global o a un modelo a escala del capital global (Foley, 1989), y 2) el sindicato no es un capitalista ni sus afiliados trabajado- 
res con los que se establezca una relación mercantil ni de explotación.

En su lugar, y dado que el interior de la estructura marxista ofrece espacio teórico para abordar un conjunto de problemáticas, se buscan referencias que orienten la interpretación. Al mismo tiempo, las distinciones teóricas no se traducen siempre en forma nítida en análisis empíricos y los problemas de clasificación no pueden resolverse de antemano (Fine y Saad-Filho, 2013). Por tanto, aquí se propone una distinción analítica que sólo puede ser comprendida acabadamente a través de una investigación empírica detallada, que en este artículo refiere al sindicato de Luz y Fuerza Capital Federal de Argentina entre 1943 y 2003.

\section{I. El nivel de complejidad}

La noción nivel de complejidad alude al tipo de relaciones sociales que median la obtención de recursos sindicales. Mientras que ciertos ingresos provienen de aportes directos, otros son el fruto de operaciones que involucran a un conjunto de relaciones e intercambios mercantiles.

Un sindicato es una organización de la clase obrera cuyos primeros recursos económicos provienen de los aportes de los trabajadores y de los empresarios. Se trata de transferencias directas de dinero, por lo que estas son consideradas mecanismos más simples e inmediatos. En otros casos, los mecanismos que permiten la obtención de recursos se complejizan, pues por regla general comprenden procesos en que intervienen una mayor cantidad de estructuras y mediaciones sociales, es decir, ya no únicamente son los trabajadores y los empresarios quienes aportan dinero al sindicato en una operación de simple transferencia sin valor agregado, sino que ese dinero tiene mayor circulación y se vuelca a otras estructuras, como pueden ser cooperativas o emprendimientos de negocios, o bien, se producen intercambios mercantiles, relaciones de compra-venta, en 
que los objetivos y funciones otorgadas al dinero pueden diversificarse (el mismo puede estar destinado al consumo -M-D-M'- o a la valorización -D-M-D'-).

De esta manera, se entiende que un gremio interviene en su dinámica reproductiva en múltiples relaciones con diferentes grados de complejidad. Según queda evidenciado en el análisis empírico, las distinciones de los circuitos y funciones del dinero no se presentan en forma clara, lo que da lugar a categorías híbridas, como la de "mini-circuito", dentro del sistema de crédito (Harvey, 1990). Se utiliza esta categoría para caracterizar las operaciones de la cooperativa de crédito ya que, si bien la misma realiza préstamos, según se verá más adelante, los intereses no son parte de la plusvalía que el capital prestado ayuda a producir, esto porque los préstamos están destinados al consumo de los trabajadores.

Atendiendo a esta dimensión, se han clasificado los mecanismos reproductivos según los distintos niveles de complejidad de las relaciones sociales que median la obtención de ingresos.

\section{2. Los efectos sobre la estructura organizativa y patrimonial del gremio}

Los mecanismos de reproducción material pueden ser analizados también en función de sus efectos, expansivos o contractivos, sobre la estructura organizativa y patrimonial de un gremio en determinado momento histórico.

Analizar los efectos supone tener en cuenta la dinámica de los determinantes (empleo, salarios, negociación colectiva) que permiten que los distintos mecanismos puedan cumplir con su función reproductiva. La expansión o contracción de esos determinantes tendrá un efecto análogo sobre los ingresos que generen tales mecanismos.

Así, los mecanismos de reproducción material pueden tener efectos expansivos si, impulsados por los determi- 
nantes, generan un crecimiento de los ingresos, lo que se cristaliza en la estructura organizativa y patrimonial de la organización, o contractivos si lo impiden.

El análisis se complejiza al incorporar la perspectiva de las funciones del dinero en los distintos circuitos que el mismo recorre. Existe la posibilidad real de que la organización sindical active mecanismos que se independicen de aquellos determinantes y cuyo fin inmediato sea la valorización. En este caso, la expansión patrimonial no se traducirá en la ampliación del fondo de consumo gremial (hoteles, campos de recreo, etc.), sino en la conformación de unidades de negocios (empresas privatizadas, administradoras de fondos previsionales, etc., como sucedió en los años noventa).

Por lo tanto, de acuerdo a este criterio, puede evaluarse en cada periodo histórico si los mecanismos son expansivos o contractivos y si esos movimientos encuentran su fundamento en el consumo o en la valorización.

\section{3. La relación con la economía política de los trabajadores}

Por último, el análisis de la relación entre los mecanismos reproductivos de un gremio y la economía política de los trabajadores se basa en la propuesta que hace Michael Lebowitz (2005) en su libro Más allá de El Capital. La economía política de la clase obrera en Marx, acerca de reponer el ciclo de producción del trabajo asalariado en el estudio del capital como un todo, en el supuesto de que los sindicatos son fundamentales en este ciclo.

Lebowitz argumenta que El Capital es una obra epistemológicamente incompleta, ${ }^{2}$ ya que según él Marx omite analizar el proceso de producción del trabajo asalariado,

2. La idea de que El Capital es una obra incompleta, o que se completó reformulando el plan original, también se encuentra en Negri (200 I), Rosdolsky (1989), Grossman (I979), Rubel (2003) y Arthur (2006). El aporte de Lebowitz (2005) reside en reponer la centralidad de la lucha de clases por el lado de los obreros. 
el cual constituye un segundo ciclo diferente al de producción del capital. En él, el trabajador consigue artículos de consumo para satisfacer sus necesidades en la esfera de la circulación y los aniquila en el proceso de su propia producción para luego regresar a la esfera de la circulación como vendedor de fuerza de trabajo.

Ambos procesos de producción -el del capital y el del trabajo asalariado- son opuestos, se excluyen entre sí y es esta oposición excluyente la que da significado a la lucha o, en términos de Lebowitz, al enfrentamiento entre dos economías políticas, la del capital y la del trabajo asalariado. Tanto el capitalista como el trabajador tienen como objetivo la reproducción ampliada: el primero busca el aumento de la plusvalía, el segundo el crecimiento del valor de uso, es decir, la satisfacción de crecientes necesidades espirituales y sociales.

La lucha de clases es lo que determina el grado en que los trabajadores logran satisfacer sus necesidades. Esa lucha se expresa en múltiples dimensiones. Una de ellas busca impedir que se rebaje el precio de la capacidad laboral con respecto a su valor, lo que se presenta al menos en dos planos: uno refiere al incremento de los salarios, lo que implica mayores ingresos para satisfacer en forma individual las necesidades producidas por el capitalismo, y otro a la satisfacción colectiva de necesidades como la educación y la salud. En este contexto, los sindicatos ocupan un lugar central. Si bien han proclamado metas más radicales, las principales prácticas que desarrollaron en forma temprana y sostenida (la negociación colectiva y la provisión de servicios a los afiliados) los convierten en una herramienta fundamental para la satisfacción de las necesidades de los trabajadores en ambos planos de lucha.

En este marco, los sindicatos se conformaron como asociaciones solidarias dirigidas a acrecentar la satisfacción de las 
necesidades sociales de los trabajadores o, en otros términos, a expandir el ciclo de producción del trabajo asalariado.

Poner en relación los mecanismos sindicales de reproducción material con la economía política de los trabajadores implica analizar si el modo en que un gremio se sostiene económicamente se articula con su función de contribuir a la reproducción material de sus representados.

Hasta ahora, el estudio de los efectos de los mecanismos reproductivos de un gremio se orientaba a captar la eficacia de su funcionamiento en términos económicos, en tanto que los mismos le permiten obtener ingresos. En este punto, en cambio, se pretende evaluar la relación de tales mecanismos con la lucha por la expansión del ciclo reproductivo del trabajo asalariado.

De este modo, cabe hablar de mecanismos más articulados con la economía política del trabajo asalariado si su funcionamiento se dirige a ampliar al mismo tiempo el consumo de los trabajadores, o menos articulados si está disociado de tal objetivo. En este último caso, se presenta la posibilidad de que los mecanismos reproductivos del gremio estén más ligados a la economía política del capital, es decir, aquella que tiene como fin la valorización y que se expresará en el fenómeno del sindicalismo empresarial.

Estas tres dimensiones permiten caracterizar a los mecanismos de reproducción material de una organización sindical, y la primacía de los distintos mecanismos con sus características distintivas marcará cuáles son las etapas que recorre la vida material de un gremio (si estos son simples o complejos, con efectos expansivos o contractivos, articulados o no a la economía política del trabajo asalariado).

Como se ha señalado, se considera que la validez de esta propuesta sólo puede ser estimada en el análisis empírico, por lo cual se ha propuesto estudiar el caso de LYFCF de Argentina en distintas etapas. 


\section{Continuidades y transformaciones en el aspecto} económico de un gremio

Luz y Fuerza Capital Federal constituye el mayor sindicato de trabajadores de la energía eléctrica, un sector que es fundamental para el desarrollo económico por su capacidad de potenciar las fuerzas productivas y contribuir a la producción de plusvalía. Esto hace que los trabajadores eléctricos alcancen niveles salariales y convenios colectivos superiores a los de la media de la clase, constituyéndose así en una suerte de aristocracia obrera.

El lugar preeminente de LYFCF dentro del sector, y por tanto de la federación nacional, obedece a que representa a los trabajadores de las más importantes empresas eléctricas, tiene el mayor número de afiliados y negocia los mejores convenios por fuera del convenio colectivo de trabajo (CCT) nacional, hecho que le otorga una autonomía de la que otros gremios carecen.

$\mathrm{Al}$ mismo tiempo, el sindicato lucifuercista ocupa a partir de los años cincuenta un lugar protagónico al interior del movimiento obrero argentino como modelo de un tipo de sindicalismo de servicios, basado en una inmensa estructura destinada a proveer servicios sociales a sus afiliados, lo que ha sido expresión del poder económico que alcanzó gracias al funcionamiento expansivo de un conjunto de mecanismos reproductivos.

Tales mecanismos de reproducción material no siempre han sido los mismos, sino que fueron asumiendo distintas características, lo que permite delimitar los cortes o periodos de análisis.

\section{I. 1943-1976. La expansión material}

Entre 1943 y 1976, LYFCF puso en funcionamiento diversos mecanismos de reproducción material con creciente complejidad. Entre los primeros, y más simples, se ubican 
la cuota social, un aporte solidario de los trabajadores afiliados al gremio, y el aporte extraordinario a la firma de cada nuevo convenio, una porción de la masa salarial total deducida de los ingresos de los trabajadores.

Los primeros estatutos de LYFCF establecieron una cuota mensual nominal (ARS 1.00 en 1943 y 1947, ARS 2.00 en 1950), y luego el estatuto de 1958 determinó porcentualmente el aporte sobre los salarios, lo cual le permitía al sindicato actualizar sus ingresos de acuerdo a la dinámica de la negociación colectiva. En ese año, la cuota se fijó en el 4.5 por millar del salario correspondiente a la categoría D del convenio con diez años de antigüedad, y en 1974 se elevó al 10 por millar (1\%).

La cláusula según la cual los trabajadores debían efectuar un aporte extraordinario al sindicato por la firma del convenio apareció por primera vez en el CCT de 1948 con carácter voluntario, y a partir de 1958 se transformó en obligatorio. De esta manera, el gremio pasó a contar -tras la firma de cada convenio- con toda la masa de incrementos salariales de los trabajadores representados.

Fijados los porcentajes, la magnitud del dinero obtenido en virtud de estos mecanismos obedeció a un conjunto de determinantes: la cantidad de afiliados y de trabajadores bajo convenio (ligados ambos a la evolución del empleo), los salarios y la dinámica negociadora.

En esta primera etapa, además de incrementarse los porcentajes, hubo un movimiento general ascendente de los determinantes, lo que hizo que el dinero recibido por la organización experimentara un importante crecimiento. El empleo y la dinámica negociadora son los determinantes que mejor explican hoy día la evolución de los recursos captados por el gremio, dado que los salarios reales sufrieron un comportamiento oscilante.

Con respecto a la evolución del empleo, aunque se carece de series completas de datos es posible estimar que la misma 
se asemeja a la de la cantidad de afiliados, considerando que LYFCF cuenta históricamente con altos niveles de asociación. En diciembre de 1943 tenía trescientos socios efectivos; en el mismo mes de 1944 contaba con 3 800; en diciembre de 1945, 8 600, y en junio de 1946, 11500 (Dinamis, 1946). Veinte años después, el secretario de Hacienda declaraba administrar el aporte de aproximadamente 28500 afiliados activos (Dinamis, 1965). Por último, para 1975 el dirigente Juan José Taccone informaba que los afiliados de LYFCF eran 30000 (Taccone, 1976).

En relación a la negociación colectiva, entre 1945 y 1975 LYFCF renegoció escalas salariales en trece oportunidades, dinámica que impulsó la recaudación del gremio vía aportes extraordinarios del total de los trabajadores representados.

Por último, con respecto a la evolución salarial, si bien las escalas implicaron un aumento nominal, el comportamiento no fue idéntico en términos reales. Por el contrario, la evolución salarial deflactada por el índice de precios tuvo una leve tendencia decreciente (del 8\% entre 1945 y 1975), aunque en el corto y mediano plazo experimentó fuertes oscilaciones (Haidar, 2015c).

Los aportes de los empresarios fijados convencionalmente para formar los fondos sociales representaron los mayores ingresos para la organización. Estos aportes también constituyen una relación social simple sostenida en la transferencia directa de dinero al sindicato, y al ser el monto de ese dinero un porcentaje de la masa salarial total, los determinantes son una vez más el empleo y los salarios.

El mayor peso de estos ingresos en la economía gremial obedece a que mientras que las cuotas sociales tuvieron un porcentaje comparativamente menor y los aportes extraordinarios sólo se retenían una vez (al momento de negociar), los aportes de los empresarios experimentaron un crecimiento progresivo de su porcentaje (desde el $2 \%$, en 1958, al $7.5 \%$, en 1975 , destinados a residencia de vacaciones, vivienda, 
fondo compensador, cultura, educación y deporte), y su percepción era mensual.

La enorme cantidad de dinero que conformaba los fondos sociales implicó la posibilidad cierta de ponerlo en circulación en circuitos más complejos. Uno de los más significativos fue el depósito bancario a cambio de intereses, lo que permitía obtener mayores recursos y evitar los efectos devaluatorios de la inflación. Otro circuito, contenido dentro de la propia funcionalidad de los fondos sociales, se organizó en torno a la venta de mercancías a los afiliados.

En esta dinámica, las cooperativas aceitaron la circulación del ahorro obrero en operaciones que implicaron intercambios mercantiles, aunque dentro de límites estrictos. La cooperativa de consumo - por medio del llamado autoservicio sindical-vendía mercancías que eran consumidas por los trabajadores en forma directa (alimentos) o como instrumentos de consumo (electrodomésticos, materiales para la construcción, etc.).

La de seguros, por su parte, era heredera de las funciones que habían asumido las primeras organizaciones mutuales respecto a la cobertura de riesgos (incendio, robo, fallecimiento). En este caso, la venta de pólizas se dirigía no sólo a los trabajadores, sino también a los empleadores del sector, que quedaban comprometidos a lo acordado en los convenios colectivos (el seguro de vida estaba reservado por convenio a la cooperativa).

La cooperativa de vivienda, por su parte, realizaba préstamos a los afiliados, a cambio de intereses, para comprar terrenos y materiales y construir viviendas, mientras que la de créditos completaba esta función otorgando a los trabajadores créditos cuyo destino era mayormente el consumo de mercancías vendidas por el propio sindicato.

En ambos casos, las cooperativas comprometían los créditos con valores de uso específicos, lo que hace que estas estructuras, aun la crediticia, no sean homologables al 
capital a interés. Su carácter asociativo, el hecho de que los créditos se dirijan a un grupo limitado de consumidores y que los intereses cobrados estén por debajo de la media dificultan igualmente esa definición. Más apropiado, en cambio, resultaría interpretar a las cooperativas que otorgan créditos como un mini-circuito (Harvey, 1990), entendido como aquel en que el pago de intereses no constituye una porción directa de plusvalía que el capital prestado ayuda a producir, sino que conecta a una unidad económica que tiene excedentes (el sindicato) con otras que lo necesitan para diversos fines (los trabajadores).

Lo distintivo de la cooperativa de crédito es que fue interviniendo en forma progresiva en arreglos financieros más sofisticados y agregó a sus operaciones comunes otras que involucraban el manejo de valores ficticios, es decir, no respaldados por una transacción real de mercancías. En este orden se encuentra la adquisición de acciones en bolsa y de títulos públicos en nombre de los trabajadores.

La magnitud de los ingresos de las cooperativas se fue apartando así de los determinantes que la afectaban en las relaciones sociales más simples, pero esa distancia no significó total independencia, puesto que el funcionamiento de estas estructuras estaba vinculado a la negociación colectiva (como en el caso de la cooperativa de seguro) y a las mismas relaciones sociales simples que contribuían a la conformación del ahorro obrero.

Los mecanismos reproductivos tuvieron un efecto expansivo sobre la materialidad de la organización, que se vio impulsada por el comportamiento positivo de los determinantes. Dicha expansión encontró su fundamento en el consumo, que a su vez se reflejó en la evolución del patrimonio que para mediados de los años setenta llegó a estar conformado por ocho hoteles, ocho campos de recreo, cuatro jardines de infantes, el hogar del niño, el hogar de jubilados, ocho cine-teatros, el autoservicio, la imprenta 
sindical, la biblioteca, además de los centros educativos de nivel secundario y de formación profesional (Dinamis, 1973).

Al mismo tiempo, los mecanismos no implicaron solamente un mayor consumo de la organización, sino que ese proceso se desarrolló vis a vis con la ampliación del ciclo de la economía política del trabajo asalariado o, en otros términos, la reproducción ampliada de los trabajadores, es decir, aquel proceso por el cual estos trabajadores aumentan sus posibilidades de consumo.

En principio, los convenios beneficiosos para los trabajadores suponían al mismo tiempo más ingresos para el consumo directo del sindicato que, por lo demás, constituía una forma de propiedad colectiva (con la posesión de hoteles, lugares de recreo, etc.). Junto a ello, la conformación de fondos sociales con base en el aporte directo de los empresarios se dirigía a incrementar el consumo obrero. Las cooperativas que se ocupaban de la compra-venta de mercancías a precios ubicados por debajo del mercado estuvieron apuntalando este proceso.

Se entiende así que uno de los rasgos más salientes de esta etapa fue la estrecha relación entre los MSRM y el ciclo productivo del trabajo asalariado, siendo que, aun cuando se complejizaron, los primeros estaban montados sobre y orientados hacia la satisfacción de las necesidades de la familia obrera. En ese sentido, se entiende que la reproducción de la organización quedaba subordinada a la de los trabajadores.

Esta conexión estaba mediada discursivamente por la concepción del "sindicalismo múltiple", según la cual el gremio debía no sólo reclamar por salarios y condiciones de trabajo, lo que se consideraba una tarea meramente reivindicativa, sino ocuparse además de las necesidades de los trabajadores y sus familias desde que nacen hasta que mueren. Con la experiencia histórica del mutualismo, el sindicato se erigió desde entonces en la entidad impulsora 
de la solidaridad y la felicidad obreras. Los aportes volvían a los trabajadores, según la fórmula sindical.

El balance del periodo indica que entre 1943 y 1976 se expandió la economía gremial de la mano de mecanismos reproductivos que asumieron las siguientes características:

- Centralidad de las relaciones sociales simples y de sus determinantes (empleo sectorial, salarios y dinámica de la negociación) en la reproducción material del sindicato;

- Creciente complejidad de las relaciones sociales, de la mano de las funciones sociales del gremio, lo que implicó la circulación del ahorro obrero, pero no la personificación de capital;

- Efectos expansivos fundados en el consumo de la organización, lo que se expresó en el crecimiento de su estructura organizativa y su patrimonio;

- Subordinación de la reproducción de la organización a la economía política de los trabajadores, en tanto los mecanismos de reproducción material de la primera estaban montados sobre y orientados a la satisfacción de las crecientes necesidades de los segundos.

\section{2. 1976-1992. La crisis material}

El golpe militar de 1976 abrió un nuevo periodo en la economía gremial, el cual se extendió durante el primer Gobierno democrático (1983-1989) y alcanzó su punto más álgido con la privatización de la empresa estatal de energía eléctrica Servicios Eléctricos del Gran Buenos Aires (SEGBA), en 1992. Durante dicho periodo se implementaron políticas que socavaron el esquema vigente hasta entonces y que, más allá de los vaivenes, condujeron a un tendencial deterioro de la lógica reproductiva del sindicato.

En esta dirección, los mecanismos simples de reproducción material y sus determinantes fueron atacados. A su vez, los mecanismos más complejos, que suponían la circulación 
del ahorro obrero constituido sobre los anteriores, también se vieron comprometidos.

En este esquema general, la particularidad de la dictadura militar residió en que no sólo fue contra los determinantes de los mecanismos, sino que los cortó de manera directa. Desde este dato podemos leer la suspensión de las afiliaciones aplicada en el marco del decreto 385/77, que anulaba contribuciones compulsivas (como las cuotas extraordinarias) y derogaba una disposición anterior según la cual las cuotas sindicales alcanzaban a todos los trabajadores. Tras este intento de atacar las bases mismas del gremio, en abril de 1981 se redujo un cien por ciento el importe correspondiente a la cuota sindical (Cronista Comercial, 1992).

Así, se apuntalaba la desfinanciación de la organización, que tuvo como principal herramienta el ataque al convenio colectivo de trabajo, producido en febrero de 1977, con la implementación de la Ley 21.476/76, que anulaba todas las cláusulas que estuvieran por encima de lo dispuesto en la Ley de Contrato de Trabajo. Ello supuso la ampliación de la jornada laboral y la anulación de conquistas históricas como las bonificaciones, el régimen de vacaciones y los aportes al gremio.

Estas medidas fueron resistidas duramente por los trabajadores, pero tras la desaparición forzada del secretario general Oscar Smith, el sindicato fue derrotado y perdió las cláusulas convencionales que hasta entonces le habían permitido obtener un enorme caudal de ingresos. La anulación, primero, y después la disminución de la cuota de afiliación, la eliminación de los aportes extraordinarios y empresarios, la pérdida de control sobre el fondo compensador y la inactividad en la que cayeron las cooperativas significaron una brutal caída de recursos para la organización.

Al mismo tiempo, junto con el ataque a tales mecanismos, se erosionaron sus determinantes, con lo cual quedó 
comprometido el funcionamiento presente y futuro de la organización. Mientras que en 1974 la dotación de SEGBA era de 25962 trabajadores, en 1984 pasó a 21 336, un 18\% menos, descenso que puede explicarse por las cesantías producidas en virtud de la ley de prescindibilidad (Senén González y Bosoer, 2009; Rodríguez, 2012) y la reorganización empresarial. Asimismo, a nivel macroeconómico, entre 1976 y 1982 los salarios reales fueron en promedio 32.8\% más reducidos que los de 1974, esto debido a la fuerte devaluación de la moneda, el llamado sinceramiento de los precios y el congelamiento de los salarios (Arceo, et al., 2008).

Con el primer Gobierno democrático, en lo que constituyó una suerte de interludio en la tendencia general del declive económico para el sindicato, hubo una recuperación parcial de sus mecanismos reproductivos y de los determinantes que había estructurado el periodo previo, lo que sin embargo no fue suficiente para alcanzar los niveles previos a la dictadura. En este orden se puede situar el crecimiento del número de empleados en SEGBA, que pasó de 20135 en 1983 a 22269 en 1989 (Dinamis, 1991), aumento que implicó el incremento de afiliados activos de 17401 a 19582 en el mismo lapso, según datos de la Secretaría de Organización de LYFCF. A su vez, los aportes extraordinarios no fueron restituidos y los de los empresarios, si bien restablecidos en el convenio, se ubicaron en el $4 \%$, es decir, por debajo del porcentaje alcanzado en el momento de auge de la economía gremial.

Los mecanismos de mayor complejidad que se habían desarrollado sobre los más simples se vieron igualmente afectados. En ese fenómeno se ubican la desfinanciación de la caja compensadora, motivada por el resentimiento de la relación activos-pasivos (en la primera etapa era de 6 a 1 , mientras que en 1987 pasó casi a 1 a 1) (Dinamis, 1987), y la merma de la actividad cooperativa, que además de ver afectado el número de asociados perdió la prioridad de con- 
tratación que había obtenido en los convenios de los años setenta. Contraídos los ingresos de los fondos solidarios, se interrumpió la posibilidad de ponerlos en circulación.

El proceso de crisis encontró su punto más alto en 1992 con la privatización de SEGBA. La empresa pasó de tener 22809 trabajadores en 1989 a 6867 en 1998 (Duarte, 2001), descenso que continuó hacia el 2002, según puede estimarse a partir de los datos de Edesur. ${ }^{3}$ Esta distribuidora tenía, en 1992, 7541 empleados efectivos, 5051 en 1993 y 2251 en 2002 (Ministerio de Economía de la Nación, s/f; Edesur, 2002), lo que representa una reducción del 70\% en diez años.

Esta contracción implicó la caída del número de afiliados activos, que pasó de 19582 en 1989 a 3986 en 2002, y con ello una drástica reducción de dinero, al tiempo que los aportes de los empresarios, si bien experimentaron un crecimiento del porcentaje del $4 \%$ al $6 \%$, no lograron equilibrar el drenaje de recursos que supuso la contracción del empleo efectivo (que demostró ser el determinante más importante en la evolución de los ingresos gremiales).

Sumado a ello, y como consecuencia de la privatización, el sindicato asumió el control de la obra social ${ }^{4} \mathrm{y}$ del fondo compensador, institutos que en el contexto de inversión de la relación entre activos y pasivos (para 1992 había 1.5 pasivos por cada activo), lejos de generar nuevos ingresos, se tradujeron en déficit sostenido.

Siguiendo la tendencia del periodo, las cooperativas, desfinanciadas, se convirtieron en estructuras intermediarias en

3. Dado que se carece de datos para las siete empresas de electricidad en que se dividió SEGBA, se toma a Edesur como caso testigo. Se trata de la compañía con mayor número de empleados y que encabezó las nuevas políticas de recursos humanos en el sector.

4. Mientras existió SEGBA, el sindicato no tuvo una obra social propia, es decir, una prestadora de salud. Esta institución constituyó en Argentina una de las principales cajas que manejan las organizaciones gremiales. Hasta que la empresa se privatizó, en 1992, LYFCF fundó la Obra Social de los Trabajadores de las Empresas de Electricidad (OSTEE). 
la compra-venta de mercancías, y recuperaron la prioridad de contratación en los convenios, lo que, sin embargo, no parecía ser suficiente para revertir la crisis. El mensaje fue afrontar la reforma con pragmatismo, lo que LYFCF tradujo en la negociación de paliativos, como las indemnizaciones para los trabajadores eléctricos que aceptaban los retiros o la permanencia en la obra social de los tercerizados.

El ataque a los mecanismos reproductivos tuvo un efecto contractivo que se expresó en la reducción de la estructura y los gastos corrientes de la organización. Así, se pasó del abandono de las instalaciones por parte de la intervención militar a su recuperación en el Gobierno de transición a la democracia y luego, con la privatización, a una reducción de la estructura organizacional y la concesión de los hoteles adquiridos en la etapa anterior.

Asimismo, debe señalarse que a pesar de ello se mantuvo la articulación y subordinación de la reproducción del sindicato respecto al ciclo de la economía política del trabajo asalariado, sólo que la tendencia viró de la expansión a la contracción. Como la reproducción del sindicato continuó ligada a los determinantes que afectaban en primer lugar a los propios trabajadores -el empleo y los salarios-, la erosión de los mismos implicó también una disminución de los ingresos y el consumo gremial, lo que generó a su vez una caída de la circulación del ahorro del pasivo en la compra-venta de mercancías o en los préstamos de dinero a los obreros.

Una manifestación de este proceso de contracción de los ingresos de los trabajadores vis a vis con los ingresos del sindicato fue el deterioro de la obra social, que significó tanto un déficit para la organización como un perjuicio para los trabajadores, quienes por primera vez comenzaron a pagar aranceles por prestaciones anteriormente gratuitas. Ante la crisis, el sindicato ajustó sus gastos y, junto con ello, los recursos destinados a satisfacer las necesidades sociales de 
los trabajadores, a quienes dejó a expensas de las fuerzas del mercado bajo las reglas generales del capitalismo.

Este fue un punto de inflexión en un proceso de crisis más amplio de la economía gremial, que comenzó en 1976 y que tuvo las siguientes características principales:

- Erosión de las relaciones sociales simples y de los determinantes que permitían la reproducción material del sindicato (empleo sectorial, salarios y dinámica de la negociación);

- Erosión, por tanto, de las relaciones sociales complejas que se desarrollaban sobre el ahorro obrero, lo que implicó la restricción de las funciones sociales de la organización;

- Efectos contractivos en el consumo del gremio, lo que significó la reducción de la estructura organizativa y el sostenimiento del patrimonio, en algunos casos por medio de su mercantilización;

- Subordinación de la reproducción de la organización a la economía política de los trabajadores, donde la contracción de la primera estuvo dada por y amplió la contracción de la segunda.

\section{3. 1992-2003. La expansión capitalista: el "sindicalismo empresarial" 5}

Para afrontar la severa crisis económica atravesada, el gremio capitalino impulsó dentro de la federación nacional una solución que no se limitaba al ajuste de gastos, sino que permitía generar nuevas fuentes de ingresos: la estrategia denominada sindicalismo empresarial, que consistió en la participación activa en las reformas de mercado, como las privatizaciones de empresas públicas y del sistema de seguridad social.

5. Un tratamiento detenido sobre el sindicalismo empresarial, con una revisión de sus principales interpretaciones y rasgos distintivos, se encuentra en Haidar (2015a y 2016). 
LYFCF, si bien realizó una incursión menor en la adquisición de unidades de negocio, impulsó la iniciativa y negoció su aprobación dentro de la FATLYF, al tiempo que aportó su patrimonio como garantía.

El sindicalismo empresarial fue interpretado desde las producciones académicas como una deriva más o menos natural del modelo y estilo sindical dominante en Argentina. Para algunos investigadores, constituyó un esfuerzo por sostener un esquema organizativo de carácter corporativo y burocrático (De la Garza, 2001; Novick, 2001); para otros, significó el producto de un espacio de negociación con el Gobierno que fortaleció los recursos organizativos (Murillo, 1997; Etchemendy, 2001); mientras que para una tercera línea fue la prolongación de un tipo clásico de sindicalismo de servicios (Ranis, 1995; Palomino, 1999).

Estas lecturas, aun con matices, tienden a destacar los elementos de continuidad de prácticas y tradiciones previas, pero no logran captar la singularidad del fenómeno. Desde una perspectiva disímil, en este trabajo se sostiene que los emprendimientos que dieron contenido al sindicalismo empresarial (empresas eléctricas, aseguradoras de riesgos del trabajo, administradoras de fondos de jubilaciones y pensiones, etc.) constituyeron nuevos mecanismos complejos de reproducción material que venían a desplazar de su lugar central a los tradicionales, dado que, al estar en crisis, estos ponían en jaque el ingreso de dinero.

La estrategia no necesariamente fue exitosa, pero más allá de los resultados lo distintivo es que el gremio utilizó el ahorro obrero con una nueva función y objetivo: constituirse en capital y valorizarse, para lo cual montó un holding empresarial que operaba en las esferas comercial, financiera y productiva.

La iniciativa más visible dentro del circuito comercial fue la creación de dos agencias de turismo (Volt's y TurLyfe) que transformaron esta área, de cara a la tradición de acción 
social del gremio, en una mercancía lanzada a un mercado que trascendía a la familia lucifuercista, todo con el fin de obtener ganancias. Junto con ello, el sindicato intervino en forma sistemática en los circuitos del capital financiero, que estaba centrado en el sistema de crédito y la creación de formas ficticias de capital (Harvey, 1990). Habilitada por las reformas de mercado y como parte de la estrategia empresarial, la FATLYF formó una complejísima estructura de negocios financieros: la administradora de fondos de jubilaciones y pensiones (AFJP), las aseguradoras satélites y la aseguradora de riesgos del trabajo (ART).

En 1994, creó la AFJP Futura S. A. con la participación de otros gremios y del Banco UNB, cuya presencia se previó como temporal hasta que la federación formara su propio banco, ambición que no concretó. Lo notable de esta iniciativa fue la magnitud del negocio financiero que el sistema integrado de jubilaciones y pensiones constituía, un sistema de capitalización per se tal como fuera diseñado. Al 28 de febrero de 1998 la AFJP (donde la FATLYF tenía el 78\% de las acciones) había colocado cerca de ARS 200000000.00 en un porfolio de valores ficticios: acciones en sociedades anónimas, títulos públicos, fideicomisos financieros, obligaciones negociables, letras hipotecarias y títulos de fondo de inversión directa (Contacto, 1998).

El plan previsional lucifuercista incluyó además un diseño integral para que la AFJP trabajara de modo coordinado con otras dos sociedades anónimas de seguros: Fuerza S. A. y Lúmina S. A. La primera era la adjudicataria de la atención del seguro de vida e invalidez y la segunda la encargada de proporcionar servicios de renta vitalicia o jubilación programada. La ART posteriormente creada, Luz S. A., constituyó otro tipo específico de intermediación financiera, construida en este caso para atender el negocio de la salud laboral, cubriéndose así todas las aristas del sistema mercantilizado de seguridad social. 
Además de intervenir en los negocios comerciales y financieros, el gremio por primera vez en su historia participó en forma planificada y sistemática en la esfera productiva del capital, que posee la particularidad de ser la única que produce plusvalor. En este sentido, realizó algunos emprendimientos modestos, como la adquisición de un laboratorio farmacéutico (Laformed s. A.) y una droguería (Medilyf s. A.), y otros mucho más ambiciosos, como la compra, administración y fundación de empresas ligadas a la energía.

Lejos de resistir las privatizaciones, entre 1993 y 2003 la FATLYF se constituyó como copropietaria de cuatro centrales eléctricas (NOA, Sorrento, Patagónicas, Litoral), dos transportadoras (TRANSNOA, TRANSNEA) y dos distribuidoras (EJSED, EDESE); participó sin éxito en la licitación de seis empresas eléctricas (Centrales San Nicolás, Hidráulicas de Río Negro, Hidroeléctrica Diamante, Distrocuyo, EDEER, ESEBA); y por fuera de su actividad principal adquirió parte de la empresa productora de carbón YC Río Turbio y de una distribuidora de gas (GASNEA). ${ }^{6} \mathrm{Al}$ mismo tiempo, el gremio creó empresas de servicios para asistir a las compañías eléctricas.

Así, la adquisición de empresas de electricidad durante el proceso privatizador constituyó una iniciativa más de entre otras muchas formas de negocios, las cuales no sólo fueron respuestas negociadas políticamente con el Gobierno, sino que constituyeron en sí mismas mecanismos de reproducción material de carácter complejo. Lo distintivo de esta etapa es la magnitud, sistematicidad e intencionalidad que adoptó el proceso.

6.La reconstrucción realizada en este apartado de la adquisición de acciones por parte de la FATLYF fue realizada con base en lo analizado en las revistas Dinamis y Contacto, el archivo periodístico de LYFCF, la publicación Tendencias Económicas del Ministerio de Economía de la Nación e informes sobre composición empresaria provistos por el ENRE. 
En términos cuantitativos, la multiplicidad de operaciones en los circuitos de capital en que se ubicaron las diversas unidades de negocio da cuenta de las dimensiones de la estrategia empresarial, que buscó aprovechar todas las posibilidades que ofrecían las reformas a fin de captar, y hasta de producir, plusvalía. En esta línea se inscriben también la ampliación del mercado en que operaba el gremio, el cual trascendió a la familia lucifuercista para alcanzar a los consumidores en general, y el abandono de la práctica de vender mercancías a los precios más baratos del mercado.

Asimismo, desde una óptica cualitativa, la estrategia empresarial presentó además transformaciones con respecto a los mecanismos reproductivos dominantes en etapas previas, principalmente debido a que el propósito constitutivo de la identidad sindical, esto es, representar a los trabajadores y satisfacer sus necesidades, apareció mucho más distante.

La federación volcó sus recursos a la adquisición de múltiples unidades de negocios, las cuales no constituyeron un fondo de consumo destinado al disfrute de los trabajadores, sino un capital fijo constituido para captar más valor. En otros términos, la expansión no estaba fundada en el consumo, sino en la valorización.

Muchos de los nuevos mecanismos reproductivos se asentaron sobre estructuras preexistentes ligadas a los servicios sociales (como la constitución de la AFJP con base en el fondo compensador), pero estas quedaron desarticuladas respecto a los trabajadores, en tanto ya no tenían como objetivo inmediato satisfacer crecientes necesidades sociales, sino apropiarse de diferentes formas de plusvalía, distanciándose así de la lucha por la expansión del ciclo reproductivo de los trabajadores.

Las relaciones de los trabajadores con estas estructuras y su identidad con respecto al gremio se complejizaron. Además de afiliados, los dirigentes se referían a los traba- 
jadores como propietarios de las empresas, en tanto miembros de la organización, y como clientes de las unidades de negocios. Al mismo tiempo, cuando la FATLYF pasó a ser propietaria de compañías eléctricas, a la clásica relación representantes-representados se le yuxtapuso la de empleadores-empleados. En un proceso complejo, la estrategia empresarial entrañó para el gremio la contradicción de representar trabajadores y personificar capital.

Tanto la federación nacional como la filial capitalina del sindicato intentaron articular a través del discurso lo que materialmente estaba desarticulado. Desde este enfoque se lee la presentación de los emprendimientos empresariales como una expresión más del sindicalismo múltiple, operación que, como se ha sostenido en trabajos previos (Haidar, 2016), coincide con una de las interpretaciones del fenómeno en términos del business unionism, o de prolongación del sindicalismo de servicios.

En esta investigación, en cambio, se entiende que las características predominantes que asumieron los mecanismos reproductivos en esta etapa difieren cualitativamente de aquellas que primaron bajo el llamado sindicalismo múltiple, desarrollado en el primer periodo. Esquemáticamente, estas son:

- Reproducción material del sindicato autonomizada de las relaciones sociales simples y de sus determinantes (empleo sectorial, salarios y dinámica de la negociación);

- Relaciones sociales complejas que implicaron la personificación de capital;

- Constitución del patrimonio en capital, con la intención de generar efectos expansivos fundados en la valorización;

- Desarticulación entre la reproducción de la organización y la economía política de los trabajadores, en tanto los mecanismos de reproducción material de la primera 
estaban disociados de la satisfacción de las crecientes necesidades de los segundos.

Este fue un proceso complejo que estuvo atravesado por múltiples matices y contradicciones, pero más allá de las mismas y del grado de eficacia de la estrategia, es posible reconocer la existencia de una tercera etapa que se ha caracterizado como de expansión capitalista del sindicato, esto es, de acercamiento a la economía política del capital, aquella que tiene como fin la valorización y que implica una distancia de la lucha por la ampliación de la reproducción del trabajo asalariado.

\section{Conclusiones}

Este trabajo se dirigió a introducir dentro del campo de estudio del sindicalismo una perspectiva que amplíe y complejice las producciones existentes, incorporando la dimensión de la economía gremial, bajo el supuesto de que la misma es central para entender las dinámicas y estrategias de los sindicatos.

Para ello, se propuso utilizar el concepto de mecanismos sindicales de reproducción material, y analizar las continuidades y transformaciones históricas de las tres dimensiones que lo componen (el nivel de complejidad, los efectos sobre la estructura organizativa y patrimonial del gremio y la relación con la economía política de los trabajadores), todo esto en el caso del sindicato Luz y Fuerza Capital Federal de Argentina.

En cuanto al nivel de complejidad, se vio que entre 1943 y 1976 se fundaron las bases de los mecanismos tradicionales de reproducción material en el sindicato, los cuales se asentaron en relaciones sociales simples que implicaron la transferencia directa de dinero de los trabajadores y los empresarios al sindicato, sin que mediara un intercambio 
de mercancías. Tales mecanismos son, ordenados según su incidencia en la economía gremial: los aportes empresarios para constituir fondos sociales, los aportes extraordinarios efectuados por los trabajadores a la firma de cada convenio, y la cuota de afiliación.

Estos mecanismos resultaron centrales en virtud de que sus determinantes, fundamentalmente el empleo y la dinámica de la negociación, tuvieron un comportamiento expansivo, al tiempo que los salarios reales experimentaron movimientos oscilantes. El dinero recibido por el gremio de la mano de estas relaciones sociales alcanzó niveles que permitieron conformar un caudaloso fondo de ahorro y ponerlo en circulación en circuitos más complejos.

Por un lado, la organización lo volcó en depósitos bancarios a cambio de intereses, y por otro, en la venta de mercancías destinadas a satisfacer las necesidades sociales de los afiliados a través de las estructuras cooperativas, lo que llevó al sindicato a intervenir en operaciones de creciente complejidad en la esfera comercial y financiera. Esta dinámica reproductiva culminó en 1976 con el golpe de Estado militar, momento a partir del cual se abrió un periodo de crisis que se extendió durante el primer Gobierno democrático y se exacerbó con la privatización de SEGBA, en 1992.

Entre 1976 y 1992 no se crearon nuevos mecanismos de reproducción material, y aquellos que venían funcionando en el periodo anterior fueron atacados en su propia existencia o en sus fundamentos. La dictadura militar representó la primera opción, ya que dejó sin efecto los mecanismos preexistentes, y luego avanzó sobre sus determinantes. La erosión de estos últimos fue, en cambio, el camino privilegiado tras la privatización, en tanto que, si bien se sostuvieron los mecanismos reproductivos, el empleo fue atacado en forma tan brutal que los determinantes quedaron desprovistos de operatividad. Con un tercio de los trabajadores, y por tanto de los afiliados, la organización vio 
mermados dramáticamente sus ingresos y los mecanismos más complejos se vieron igualmente resentidos.

En este marco, la privatización de SEGBA no sólo afectó al gremio en sus bases de sustentación, sino que le provocó una grave crisis económica que, dadas las posibilidades abiertas con las reformas de mercado, el gremio intentó resolver por medio de nuevos mecanismos reproductivos complejos, lo que inauguró una tercera etapa en la economía sindical.

Entre 1992 y 2003 la FATLYF, promovida por LYFCF, protagonizó una estrategia reproductiva de carácter empresario. Esto implicó ubicar en un lugar subsidiario a las relaciones sociales simples y establecer circuitos más complejos que, a diferencia de los dominantes en la primera etapa, se independizaron tanto de aquellas relaciones simples como de sus determinantes. El gremio volcó el ahorro obrero en la constitución de un complejo holding empresarial, y por primera vez en su historia inició un proceso sistemático de capitalización como forma de obtener recursos.

En la primera etapa, el sindicato había desarrollado experiencias puntuales de circulación de dinero e intercambio mercantil, pero ello no había implicado su transformación en capital, ya que LYFCF operaba con límites claros: si bien vendía mercancías a los afiliados, lo hacía a los precios más baratos del mercado y teniéndolos como únicos destinatarios. Esto era así porque su finalidad era satisfacer las necesidades sociales, no obtener ganancia ni interés. Hacia el tercer periodo, en cambio, la FATLYF desarrolló un plan de capitalización que supuso trascender los límites a los que antes el sindicato estaba sujeto, e intervino en la esfera financiera, comercial y productiva, con el fin de captar y producir plusvalor.

A partir del año 2003 y hasta el 2010, la FATLYF fue desarmando el holding creado durante los diez años previos. Los motivos que llevaron a esta decisión sustentan la hipótesis de que la estrategia empresarial fue una respuesta a la 
crisis económica sufrida por la contracción del empleo y el salario. Algunas unidades de negocios no lograron competir exitosamente en el mercado, como la ART Luz S. A., que fue autoliquidada en 2006, o las compañías energéticas, que tras la crisis de 2001 resultaron con déficit y fueron vendidas. La mayor intervención del Estado también llevó al abandono de la estrategia, lo que quedó de manifiesto con la liquidación forzosa de la AFJP Futura S. A. tras la reestatización de los fondos jubilatorios dispuesta por el Gobierno en 2010.

El fracaso en la competencia de mercado y la mayor intervención estatal se enlazan a su vez con la reactivación que experimentó el sector, en sintonía con un contexto nacional de crecimiento de la economía, el empleo y la negociación colectiva. ${ }^{7}$ Los datos sobre el comportamiento de los determinantes claves, el empleo y el salario en LYFCF, muestra que experimentaron un incremento en relación a los años noventa, y que ello permitió fortalecer los mecanismos simples de reproducción material, la cuota de afiliación y los aportes empresariales para fondos sociales. El sindicato, aun con limitaciones, volvió a replegarse sobre ellos.

En cuanto a los efectos sobre la estructura organizativa y patrimonial del gremio, en el periodo que va de 1943 a 1976 los MSRM tuvieron efectos expansivos, que se cristalizaron en la ampliación del fondo de consumo de la organización: hoteles, recreos, teatros. Como permitió evidenciar la dimensión anterior, esto se debió al comportamiento positivo de los principales determinantes que sustentan las relaciones sociales simples y aquellas más complejas que se derivan de la circulación del ahorro obrero. Esta orientación expansiva modificó su sentido en el segundo periodo de la economía gremial. 
Dicho viraje respondió al ataque a los mecanismos reproductivos y sus determinantes que, si bien entre 1976 y 1992 sufrieron algunos vaivenes, redundaron en un efecto contractivo sobre la estructura organizativa y el patrimonio del gremio. Este aplicó una política de ajuste de gastos, destinó los recursos disponibles a sostener el consumo corriente y, en un acercamiento al criterio organizador de la siguiente etapa, mercantilizó parte de su estructura social, la de los hoteles. Este proceso llevó al presente trabajo a caracterizar a estos años como de crisis material del sindicato.

Por último, en el periodo que se extiende entre 1992 y 2003, la federación nacional, con el patrimonio de LYFCF como garantía y con el ahorro obrero puesto a funcionar como capital, formó unidades de negocios con el fin de generar ganancia e interés. El holding incluyó farmacias y una droguería, una administradora de fondos previsionales con sus aseguradoras de vida y de renta vitalicia, una empresa de turismo y varias compañías de energía. Los principales emprendimientos permiten identificar a la etapa como de expansión capitalista del sindicato.

Mientras que entre la fase de crisis y la de expansión existe una diferencia de carácter cuantitativo, produciéndose una reversión entre una performance positiva y otra negativa sobre idénticas bases, el sindicalismo empresarial entraña una diferencia cualitativa con respecto a aquellas fases. En los primeros periodos, el patrimonio gremial suponía un complejo social destinado al consumo obrero-sindical, mientras que en el tercero el patrimonio fue sinónimo de capital orientado a captar y producir valor.

En cuanto a la relación con la economía política de los trabajadores, entre 1943 y 1976 los MSRM y la economía política de los asalariados se encontraban estrechamente vinculados, donde los primeros estaban montados sobre y orientados a expandir la segunda. En otros términos, el gremio obtenía en forma primaria ingresos cuya magnitud 
derivaba del volumen de empleo, los niveles salariales y la dinámica de la negociación, tres factores que inciden en forma directa sobre los propios trabajadores. Con esos recursos, la organización constituyó fondos que, administrados en gran medida por las cooperativas, le permitieron expandir tanto la propia organización como el ciclo reproductivo de la familia obrera.

Detrás de esta acción, se encontraba la noción de sindicalismo múltiple, una plataforma discursiva heredera de la tradición mutualista de las primeras organizaciones obreras, según la cual el sindicato debía trascender la reivindicación económica y volcarse al aspecto social de la vida del trabajador.

En el periodo que va de 1976 a 1992, la articulación entre reproducción sindical y obrera se sostuvo, sólo que, como quedó cristalizado en la evolución patrimonial del gremio, se invirtió la tendencia de la expansión a la contracción. La organización siguió sujeta a los determinantes que incidían en primer lugar sobre los propios trabajadores; erosionados los mismos, también entraba en crisis la economía sindical, y los trabajadores vieron reducida la posibilidad de incrementar la satisfacción de sus necesidades al limitarse la acción social del gremio. Múltiples fenómenos se encuadran en este sentido: los hoteles fueron concesionados, la obra social redujo y aranceló las prestaciones, las cooperativas perdieron operatividad y la caja compensadora se disolvió.

En cambio, durante el periodo que se extiende entre 1992 y 2003, los nuevos mecanismos reproductivos del gremio ya no dependían del ciclo productivo de los trabajadores ni contribuían a expandirlo, al tiempo que los viejos mecanismos fueron refuncionalizados para potenciar el nuevo esquema.

Los vínculos materiales entre el gremio y los trabajadores se resignificaron. La mayor parte de los afiliados que en los últimos años habían ayudado a sustentar la estructura gremial se vieron despojados de su empleo; los que lo 
conservaron quedaron sujetos a una lógica mercantilista e individualizadora, algunos se convirtieron en clientes de los negocios de la FATLYF, como la AFJP y las aseguradoras, y muchos además quedaron ligados a la federación en un nuevo sentido, más precisamente, como empleados de las empresas eléctricas en las que la primera participó.

La distancia entre la reproducción del gremio y la de los trabajadores se intentó salvar con un discurso continuista que presentó a la estrategia empresarial como una prolongación de la prestación de servicios sociales, pero mientras que la concepción del sindicalismo múltiple armonizaba en términos discursivos lo que materialmente ya funcionaba en forma articulada, la reposición de este discurso para referir a una práctica disímil trataba de ligar lo que estaba disociado en su materialidad.

En la primera y segunda etapa, ambos términos (la reproducción del gremio y la de los trabajadores) estaban estrechamente vinculados, sólo que en una en un sentido expansivo y en otra contractivo. En la tercera etapa, en cambio, y aun con matices y complejidades, predominó en forma tendencial la disociación entre los mecanismos reproductivos del gremio y su función de expandir la economía obrera. Este fenómeno tensiona la identidad de los sindicatos como asociaciones solidarias dirigidas a acrecentar la satisfacción de las necesidades sociales de los trabajadores, y en el caso del gremio analizado complejizó sus prácticas y aquel sentido perdió centralidad frente a una estrategia que supuso el acercamiento a la economía política del capital.

El estudio de las continuidades y transformaciones en los mecanismos reproductivos de un gremio a lo largo de distintas etapas históricas volvió observable que el aspecto económico importa, junto con los aspectos identitarios y político-institucionales, las características de la estructura sindical y las capacidades de negociación, de representación y movilización de los trabajadores. Se ha advertido que el 
aspecto económico impacta sobre el sostenimiento y desarrollo de la propia organización: mecanismos expansivos generan idénticos efectos sobre la estructura organizacional y el patrimonio de un gremio.

Por tal motivo, la lectura sobre el comportamiento de los mecanismos reproductivos, sus posibilidades y efectos, incide en la definición de las estrategias sindicales para garantizar el funcionamiento de la propia organización. En este punto, resultó clave el análisis del sindicalismo empresarial como una salida específica a una situación de crisis que, cuando comenzó a revertirse, y dadas las limitaciones encontradas, fue abandonada.

En relación a esta problemática, se concluyó que LYFCF desarrolló en etapas previas mecanismos reproductivos cercanos a prácticas capitalistas, pero se trataron de experiencias que no fueron predominantes en el esquema de funcionamiento sindical, y que además se subordinaron a la economía obrera.

En este sentido, los argumentos de este artículo contribuyeron a evidenciar el vínculo que existe entre la economía de un gremio y la de sus trabajadores. Aquí el interés estuvo focalizado en el primer término, la economía gremial, y en torno a él fueron analizadas las relaciones con los trabajadores, quienes en una etapa fundacional ayudaron a sostenerla y se vieron materialmente beneficiados por los servicios que aquella les brindó.

Se identificó, asimismo, que los mecanismos reproductivos del gremio encontraron fundamento y soporte en una dimensión ideológica y política, según la cual entre las funciones de la organización está participar en la construcción del bienestar de los trabajadores, a través de la provisión de un conjunto cada vez mayor de servicios. Esa definición, siempre reivindicada, fue utilizada en distintos momentos para legitimar las disímiles relaciones sociales que estableció el gremio para sostener su economía. Queda pendiente 
avanzar en investigaciones que profundicen el estudio sobre la interacción entre las diferentes dimensiones de análisis.

Del mismo modo, entender que existe una relación entre economía del sindicato y de los trabajadores abre la posibilidad de introducir nuevos interrogantes alrededor del segundo término de este vínculo. Esto supone estudiar el conjunto de relaciones sociales que intervienen en la reproducción obrera.

Lebowitz (2005), por ejemplo, dio cuenta de la falta de análisis existente en torno a lo que llamó la economía política de la clase obrera: ¿cómo se reproducen los trabajadores?, ¿por medio de qué mecanismos y luchas?

Muchos estudios se ocuparon de sostener que una parte importante de la reproducción social se lleva a cabo a través de prácticas que se ubican fuera de las relaciones de producción capitalistas, por más que sean subordinadas o dependan de ellas y aunque el proceso de producción mismo reproduzca a los trabajadores y a los capitalistas como clases separadas. Sin embargo, aún está pendiente una investigación profunda que analice el lugar que ocupan en este proceso las organizaciones sindicales, más allá de su participación en la determinación del salario.

Estos interrogantes dan cuenta de que aun con las limitaciones y contradicciones que presenta el sindicalismo, su estudio continúa siendo una tarea tan necesaria como pertinente a fin de entender su supervivencia como fuerza social y de complejizar sus prácticas y funciones en el marco de la disputa entre capital y trabajo.

Abal Medina, P., y Diana Menéndez, N. (20I I). Colectivos Bibliografía resistentes. Procesos de politización de trabajadores/as en la Argentina contemporánea. Buenos Aires: Imago Mundi. Arceo, N., Monsalvo, A., Schorr, M., y Wainer, A. (2008). Empleo y salarios en la Argentina: una visión de largo plazo. Buenos Aires: Capital Intelectual. 
Bibliografía
Arthur, C. (2006). “The inner totality of capitalism”. Historical Materialism, I4, 85-III.

Battistini, O. (comp.) (2004). El trabajo frente al espejo. Continuidades y rupturas en el proceso de construcción identitaria de los trabajadores. Buenos Aires: Editorial Prometeo.

Bensusán, G. (2000). "La democracia en los sindicatos”, en E. De la Garza (coord.), Tratado Latinoamericano de Sociología del Trabajo (pp. 392-420). México: FCE.

Bunel, J. (1992). Pactos y agresiones. El sindicalismo argentino ante el desafio neoliberal. Buenos Aires: FCE.

Camarero, H. (2007). A la conquista de la clase obrera. Los comunistas y el mundo del trabajo en la Argentina, 19201935. Buenos Aires: Siglo XXI, Editora Iberoamericana.

Collier, D., y Collier, R. (I99I). Shaping the Political Arena. Princeton: Princeton University Press.

Contacto (1998).“Cartera de inversiones”. Contacto, (76), s/n.

Cronista Comercial (06 de febrero de 1992). "Juicios contra SEGBA por cuota sindical", s/p.

Cuadernos de pasado y presente (1978)."Economía y política en la acción sindical”. Cuadernos de pasado y presente. México: Siglo XXI.

De la Garza, E. (200I).“Introducción: las transiciones políticas en América Latina, entre el corporativismo sindical y la pérdida de imaginarios colectivos", en E. De la Garza (comp.), Los sindicatos frente a los procesos de transición política (pp. 9-24). Buenos Aires: CLACSO.

Del Campo, H. (1 983). Sindicalismo y peronismo. Los comienzos de un vínculo perdurable. Buenos Aires: CLACSO.

Dinamis (1946).“Administración del sindicato autónomo de Luz y Fuerza”. Dinamis, (2I), 4.

(1965).“El patrimonio del sindicato”. Dinamis, (I0I), 46.

(1973).“1943-30 años-1973”. Dinamis, (edición 30 aniversario). 
(1987). “La vida orgánica de nuestro gremio”. Dina-

Bibliografía mis, (9), 50.

(I99I). “Reseña de SEGBA”. Dinamis, (29), 42.

Doyon, L. (1984). “La organización del movimiento sindical peronista. 1946-1955”. Desarrollo Económico, 24(94), 203-234.

Duarte, M. (200I).“'Los efectos de las privatizaciones sobre la ocupación en las empresas de servicios públicos". Realidad Económica, (I82), 32-60.

Edesur (2002). Memoria, reseña informativa y estados contables al 3 I de diciembre de 2002 y 200 I juntamente con el auditor y el informe de la comisión fiscalizadora. Recuperado de: http://www.edesur.com.ar/documentos/balance02.pdf

Etchemendy, S. (200I). “Construir coaliciones reformistas: la política de las compensaciones en el camino argentino hacia la liberalización económica". Desarrollo Económico, 40(160), 675-706.

_ y Palermo, V. (1998). "Conflicto y concertación. Gobierno, congreso y organizaciones de interés en la reforma laboral del primer gobierno de Menem (19891995)". Desarrollo Económico, 37(I48), 559-590.

Falcón, R. (1984). Los orígenes del movimiento obrero (I8571899). Buenos Aires: CEAL.

Fernández, A. (2002). Sindicatos, crisis y después. Una reflexión sobre las nuevas y viejas estrategias sindicales argentinas. Buenos Aires: Biebel.

Fine, B.,y Saad-Filho, A. (20I3). El capital de Marx. México: FCE. Foley, D. (1989). Para entender El Capital. La teoría económica de Marx. México: FCE.

Gordillo, M. (199I)."Los prolegómenos del Cordobazo: los sindicatos líderes de Córdoba dentro de la estructura de poder sindical". Desarrollo Económico, 3 I (I22), I63-I87.

Grossman, H. (1979). Ensayos sobre la teoría de las crisis. Dialéctica y metodología en El Capital. México: PYP. 
Haidar, J. (2015a). "Interpreting Argentine Business Unionism”. Latin American Perspectives, 42(2), 60-73.

(20I5b). “La economía gremial, entre la crisis y la recomposición: Luz y Fuerza Capital Federal (19762013)". Polhis, (16), 69-94.

- (2015c). Continuidades y transformaciones en los mecanismos de reproducción material del sindicato Luz y Fuerza Capital Federal (1943-2003) (tesis doctoral inédita). Universidad de Buenos Aires: Buenos Aires.

- (2016). “El sindicalismo empresarial en los años 90. Una aproximación desde su materialidad". Archivos de historia del movimiento obrero y la izquierda, (8), 77-96.

Harvey, D. (1990). Los límites del capitalismo y la teoría marxista. México: FCE.

Hyman, R. (1978). El marxismo y la sociología del sindicalismo. México: Ediciones Era.

Iñigo Carrera, N. (2004). La estrategia de la clase obrera. 1936. Buenos Aires: La Rosa Blindada-Ediciones Madres de Plaza de Mayo.

James, D. (1999). Resistencia e integración. El peronismo y la clase trabajadora argentina. 1946-1976. Buenos Aires: Sudamericana.

Korpi, W. (1985). "Power Resources Approach vs. Action and Conflict: On Causal and Intentional Explanations in the Study of Power". Sociological Theory, 3(2), 3 I-45.

Lapides, K. (1987). Marx and Engels on the Trade Unions. Nueva York: Praeger Publishers.

Lebowitz, M. (2005). Más allá de El Capital. La economía política de la clase obrera en Marx. Madrid:Akal.

Lévesque, C., y Murray, G. (2004). El poder sindical en la economía mundial. Documento $N^{\circ}$ 2. Bilbao: Manu RoblesAragiz Institutua.

Levitsky, S. (2005). La transformación del justicialismo. Del partido sindical al partido clientelista, 1983-1999. Buenos Aires: Siglo XXI. 
Lipset, S. (1963). El hombre político. Buenos Aires: Eudeba. Löbbe, H. (2006). La guerrilla fabril: clase obrera e izquierda en la Coordinadora de Zona Norte del Gran Buenos Aires: 1975-1976. Buenos Aires: Ediciones RyR.

Mandel, E. (1973). Control obrero, consejos obreros, autogestión (antología). Tomo I. Buenos Aires: La Ciudad Futura. (1987). La burocracia de los sindicatos, los partidos y los estados obreros. México: Ediciones y Distribuciones Hispánicas.

Michels, R. (20I0). Los partidos politicos. Madrid:Amorrortu. Ministerio de Economía de la Nación (s/f). Personal Edesur. Recuperado de: http://mepriv.mecon.gov.ar/segba/ memybces/personal-edesur.htm

Moe, T. (1980). The Organization of Interests. Incentives and the Internal Dynamic of Political Interests Groups. Chicago: University of Chicago.

Montes Cató,J., y Ventrici, P. (2008).“Construcciones democráticas y resistencia. Delegados y prácticas políticas en las organizaciones sindicales", en P. Lenguita, y J. Montes Cató (comps.), Resistencias laborales. Experiencias de repolitización del trabajo en Argentina (pp. 87-125). México: Insumisos.

Murillo,V. (1997). “La adaptación del sindicalismo argentino a las reformas de mercado en la primera presidencia de Menem”. Desarrollo Económico, 37(147), 419-446.

Murmis, M.,y Portantiero,J. ( 1987$)$. Estudios sobre los orígenes del peronismo. Buenos Aires: Siglo XXI.

Negri,A. (200I). Marx más allá de Marx. Madrid:Akal.

Novick, M. (200I). "Nuevas reglas de juego en la Argentina, competitividad y actores sindicales", en E. De la Garza Toledo (comp.), Los sindicatos frente a los procesos de transición política (pp. 25-46). Buenos Aires: CLACSO.

Nuevo Topo (2010). “Dossier. Hacia un debate sobre el concepto de burocracia sindical”. Nuevo Topo, (7), s/p. 
Palomino, H. (1999). "Los sindicatos en la Argentina contemporánea”. Nueva sociedad, (I69), I 2 I - I 34.

Ranis, P. (1995). Challenges to Organized Labor in the PostIndustrial World: Argentina, Germany and the U. S. Nueva York: New York College and Graduate Center, CUNY, Mimeo.

Robinson, I. (19-23 de noviembre de 1998). "Neoliberal restructuring and Union Political Power in Canada" (ponencia preparada para el $/ 4^{\text {th }}$ Biennial Meeting of the Association for Canadian Studies in the U. S.). Minneapolis, Estados Unidos.

Rodríguez, J. (20I2). La mirada implacable del "Gato" Smith. Buenos Aires: Corregidor.

Rosdolsky, R. (1989). Génesis y estructura de El Capital de Marx (estudios sobre los “Grundrisse”). México: Siglo XXI editores.

Rubel, M. (2003). Marx sin mito. Madrid: Octaedro.

Schmitter, P. (1992). “Continúa el siglo del corporativismo?”, en P. Schmitter y G. Lembruch (coords.), Neocorporativismo I. Más allá del Estado y el mercado (pp. 15-66). México:Alianza Editorial.

Schneider, A. (2007). Los compañeros. Trabajadores, izquierda y peronismo. 1955-1973. Buenos Aires: Imago Mundi.

Senén González, S., y Bosoer, F. (2009). Breve historia del sindicalismo argentino. Buenos Aires: El Ateneo.

Stepan-Norris, J., y Zeitlin, M. (1995). "Union Democracy, Radical Leadership and the Hegemony of Capital". American Sociology Review, 60, 829-850.

Suriano,J. (1990). El Estado argentino frente a los trabajadores urbanos: política social y represión, 1880-19/6. Anuario $N^{\circ}$ 14. Escuela de Historia. Rosario: UNR.

Taccone, J. (1976). 900 días de autogestión en SEGBA. Una experiencia argentina de participación. Buenos Aires: Fundación 200I. 
El estudio de la economía gremial. El caso del sindicato argentino Luz y Fuerza Capital Federal (1943-2003)

Torre,J. (1990). La vieja guardia sindical y Perón. Buenos Aires: | Bibliografía Sudamericana.

Zapata, F. (2004). ¿Crisis del sindicalismo en América Latina? México: Centro de Estudios Sociológicos del Colegio de México. 